\title{
Numerical simulations of the CME on 2010 April 8
}

\author{
Yingna $\mathrm{Su}^{1}$, Bernhard Kliem ${ }^{2}$, Adriaan van Ballegooijen ${ }^{1}$, \\ and Edward Deluca ${ }^{1}$ \\ ${ }^{1}$ Harvard-Smithsonian Center for Astrophysics, Cambridge, MA, United States \\ email: ynsu@head.cfa.harvard.edu \\ ${ }^{2}$ Institute for Physics and Astronomy, University of Potsdam, Potsdam, Germany
}

\begin{abstract}
We present 3D zero-beta ideal MHD simulations of the solar flare/CME event that occurred in Active Region 11060 on 2010 April 8. The initial magnetic configurations of the two simulations are stable nonlinear force-free field and unstable magnetic field models constructed by $\mathrm{Su}$ et al. (2011) using the flux rope insertion method. The MHD simulations confirm that the stable model relaxes to a stable equilibrium, while the unstable model erupts as a CME. Comparisons between observations and MHD simulations of the CME are also presented.
\end{abstract}

Keywords. Sun: coronal mass ejections (CMEs), Sun: flares, Sun: magnetic fields.

\section{Introduction}

A GOES B3.7 two-ribbon flare occurred in NOAA Active Region 11060 around 02:30 UT on 2010 April 8. The associated CME median speed is about $510 \mathrm{~km} \mathrm{~s}^{-1}$. In our earlier paper ( $\mathrm{Su}$ et al. 2011), we constructed a series of static magnetic field models of the active region using the flux rope insertion method (van Ballegooijen 2004). We find that the axial flux of the flux rope $\left(\Phi_{a x i}=4 \times 10^{20} \mathrm{Mx}\right)$ in the best-fit pre-flare nonlinear force-free field model is very close to the threshold of instability $\left(\Phi=5 \times 10^{20}\right.$ $\mathrm{Mx})$. The unstable model $\left(\Phi=6 \times 10^{20} \mathrm{Mx}\right)$ due to increase of axial flux closely matches the observations at the early stage of the eruption. The poloidal flux in these models remains the same, i.e., $F_{\text {pol }}=10^{10} \mathrm{Mx} \mathrm{cm}^{-1}$.

\section{MHD Simulation versus Observations}

In the present study, we perform 3D zero-beta MHD simulations (Kliem et al. 2004) using the aforementioned static magnetic field models as initial configurations. The magnetic field models are constructed in spherical geometry, while the MHD simulations are carried out in a cartesian geometry. The coordinate conversion is implemented by producing scaled down (scaling factor $=1 / 16.97$ ) versions of the original magnetic models as inputs for the MHD simulations. The computation domain of the MHD simulation is $.72 \times .72 \times 1$ solar radii. The top and side boundaries are closed. The model for the initial density is $\rho(x, y, z, 0)=|B(x, y, z, 0)|^{1.5}$.

Figure 1 shows selected field lines from the two MHD simulations using the stable (top row) and unstable (bottom row) models as initial conditions. As shown in Figure 1, the MHD simulations confirm that the stable model is really stable, while the unstable model erupts as a CME. STEREO_A observations suggest that the rising path of the erupting filament is not radial, but rather inclined towards the equator by about $45^{\circ}$. This inclination is also identified in the MHD simulation of the unstable model. Figure 2 shows a good agreement between observations and simulations of the temporal evolution of the position and velocity of selected coronal loops. 

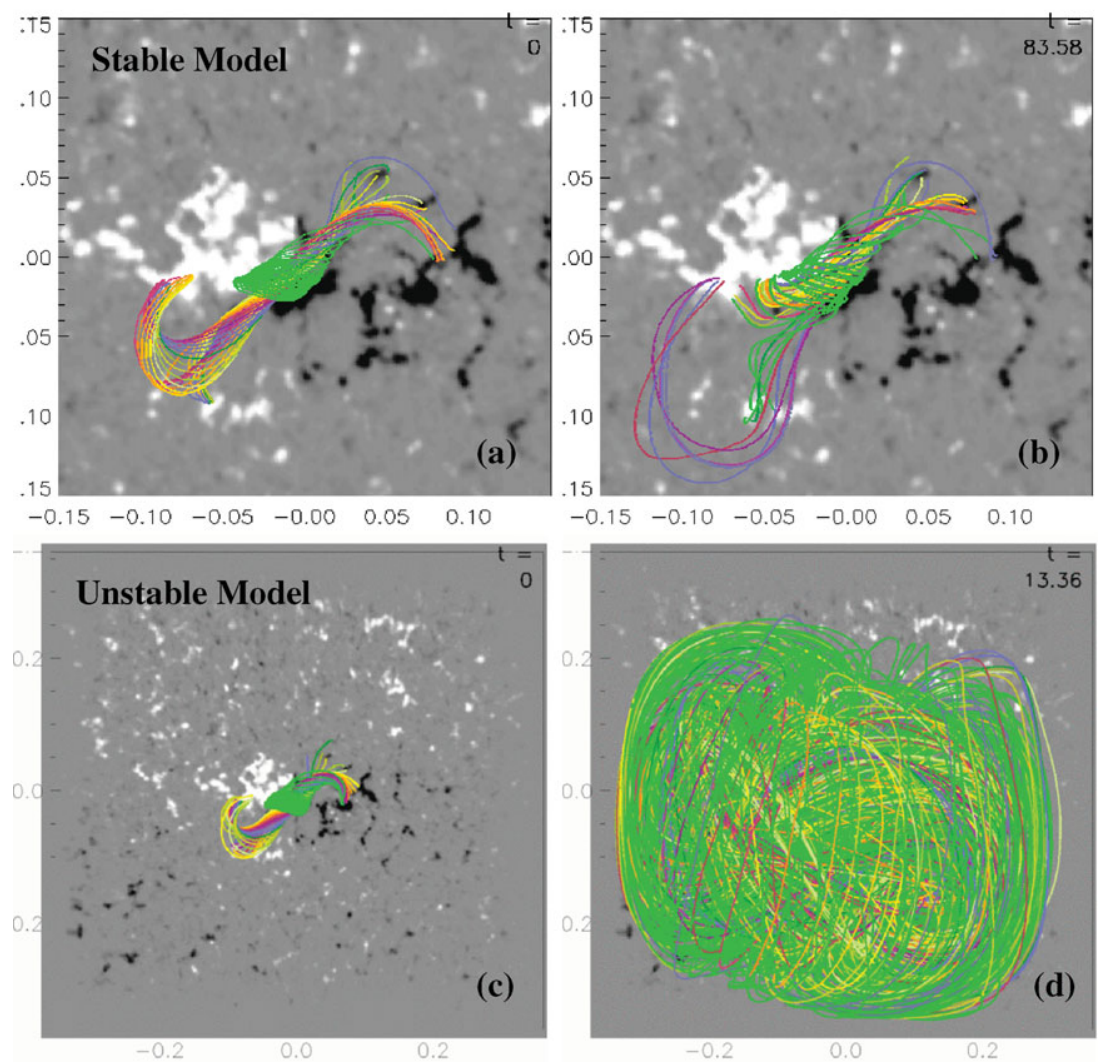

Figure 1. Initial (left) and final (right) magnetic configurations of two MHD simulations of the event. The initial conditions of the two simulations are stable (top) and unstable (bottom) magnetic field models.

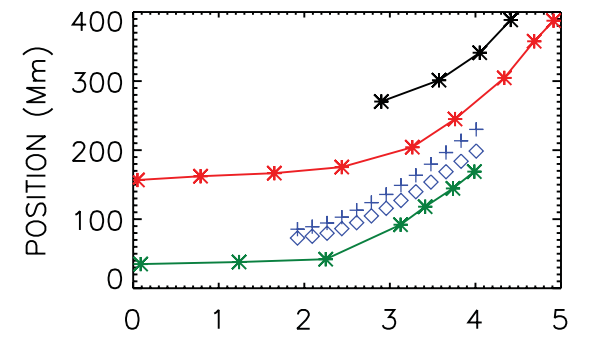

TIME (ks since 2010-04-08 02:00 UT)

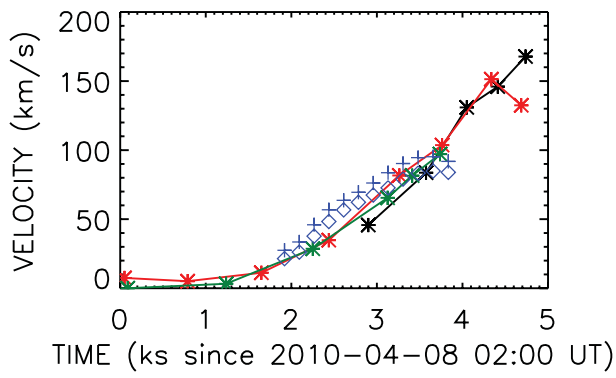

UT)

Figure 2. Comparisons between observations (asterisk) and MHD simulations (diamond and plus sign) of the temporal evolution of the position (left) and velocity (right) of selected coronal loops.

Acknowledgements: We thank the teams of SDO/HMI and STEREO/EUVI for providing the valuable data. This project is supported under contract SP02H1701R from LMSAL to SAO, by the DFG, and by the STFC.

\section{References}

Kliem, B., Titov, V. S., \& Torok, T. 2004, A $\xi A, 413$, L23

Su, Y., Surges, V., van Ballegooijen, A., DeLuca, E., \& Golub, L. 2011, ApJ, 734, 53

van Ballegooijen, A. A. 2004, ApJ, 612, 519 